\title{
Oferta de leña de los bosques nativos comunales en la cordillera del Vilcanota, Cusco, Perú
}

\author{
Offer of firewood from the comunal native forests \\ in the Vilcanota mountain range, Cusco, Peru
}

\author{
Juan Eduardo Gil-Mora ${ }^{1, \star}$, Modesta Esther Álvarez-Moscoso², \\ Graciela Sánchez-Valenzuela ${ }^{3}$ y Jesús Ángel Baca-Flores ${ }^{4}$
}

Recibido: 19 agosto 2020 | Aceptado: 09 noviembre 2021 | Publicado en línea: 29 diciembre 2021 Citación: Gil-Mora, JE; Álvarez-Moscoso, ME; Sánchez-Valenzuela, G; Baca-Flores, JA. 2021. Oferta de leña de los bosques nativos comunales en la cordillera del Vilcanota, Cusco, Perú. Revista Forestal del Perú 36(2): 211-246. DOI: http://dx.doi.org/10.21704/rfp.v36i2.1799

\begin{abstract}
Resumen
Diversos factores han contribuido a la pérdida y degradación de los bosques andinos, especialmente de los altos Andes por encima de los $3200 \mathrm{~m}$ de altitud, donde se desarrolló el presente trabajo; el ámbito de estudio se halla en la cordillera del Vilcanota que es uno de los ramales meridionales de la cordillera oriental de los Andes; la importancia de los ecosistemas forestales andinos radica primordialmente en la relación del bosque como medio natural (importancia ecológica) y también en la relación del bosque como uso y aprovechamiento por las poblaciones (importancia económica). Los bosques en los altos Andes son residuales y se hallan sometidos a una extracción constante debido a que ofertan leña, madera y otros servicios ecosistémicos; por lo tanto, tienen importancia en la economía familiar de las comunidades campesinas altoandinas del Cusco y, desde hace décadas se observa la creciente escasez de leña para uso doméstico. El objetivo del trabajo fue evaluar la oferta de leña que brindan los bosques nativos en los altos Andes; se evaluaron 18 bosques nativos comunales pertenecientes a 11 comunidades campesinas y a dos provincias (Calca
\end{abstract}

\footnotetext{
${ }^{1}$ Escuela de Posgrado, Universidad Andina del Cusco (UAC), Cusco, Perú.

${ }^{2}$ Escuela Profesional de Biología; Facultad de Ciencias, Universidad Nacional de San Antonio Abad del Cusco (UNSAAC), Cusco, Perú.

${ }^{3}$ Consultor independiente.

${ }^{4}$ Escuela Profesional de Física, Facultad de Ciencias, Universidad Nacional de San Antonio Abad del Cusco (UNSAAC), Cusco, Perú.

*Autor de Correspondencia: $\underline{\text { mundoandino2005@yahoo.es }}$
} 

en la cordillera del Vilcanota, Cusco, Perú

y Urubamba) cuya superficie total es 764 ha con una oferta total de leña equivalente a 169699,71 t; la tipología de los bosques identificados pertenece a bosque mixto arbóreo, matorral espinoso y bosque de Polylepis. Para el análisis, la zona de estudio fue dividida en dos zonas y para la obtención de la información se utilizaron transectos lineales para el matorral espinoso y parcelas cuadradas para el bosque mixto y el de Polylepis; para la determinación del volumen de leña ofertada por los bosques en campo se midieron la circunferencia basal y distal y, la longitud del fuste, y se emplearon métodos experimentales de laboratorio.

Palabras clave: bosque nativo, humedad, madera, matorral, servicios ecosistémicos

\begin{abstract}
Various factors have contributed to the loss and degradation of Andean forests, especially in the high Andes above $3200 \mathrm{~m}$ of altitude, where the present work was carried out; the scope of study is in the Vilcanota mountain range, which is one of the southern branches of the eastern Andes mountain range, the importance of Andean forest ecosystems lies primarily in the relationship of the forest as a natural environment (ecological importance) and also in the relation of the forest as use and exploitation by the populations (economic importance). The forests in the high Andes are residual and are subject to continuous extraction because they offer firewood, wood and other ecosystem services; therefore, they are important in the family economy of the high Andean peasant communities of Cusco and, for decades, the growing shortage of firewood for domestic use has been observed. The objective of the work was to evaluate the supply of firewood provided by native forests in the high Andes; 18 communal native forests belonging to 11 peasant communities and two provinces (Calca and Urubamba) whose total area is 764 ha were evaluated with a total supply of firewood equivalent to 169699,71 tons; the typology of the identified forests belongs to mixed arboreal forest, thorn scrub and Polylepis forest, for the analysis, the study area was divided into two zones and to obtain the information, linear transects were used for the thorn scrub and square plots for the mixed forest and that of Polylepis; to determine the volume of firewood supplied by the forests, the basal and distal circumference and the length of the stem were measured in the field, and were used experimental laboratory methods.
\end{abstract}

Key words: ecosystem services, moisture, native forest, scrub, wood

\section{Introducción}

Los bosques andinos son formaciones de gran importancia en el país por su alta diversidad de especies y alto grado de endemismos. Se distribuyen de manera heterogénea en el territorio peruano, y particularmente, los bosques montanos altoandinos como su nombre lo indica, son aquellos que se encuentran ubicados en las zonas más altas de los Andes, donde factores ambientales y humanos han condicionado su distribución a pocas y pequeñas zonas de laderas, quebradas y valles (Young y León 2001).

De acuerdo con Otárola (2008), la deforestación en los bosques andinos está asociada principalmente a la actividad agrícola de dife- rente escala y a la ganadería, y, en menor medi$\mathrm{da}$, a la tala de madera y a otros factores (tales como desarrollo de infraestructura, incendios forestales, proyectos hidroeléctricos, colecta de leña, etc.). Los bosques de neblina de la vertiente oriental de la cordillera de los Andes son ecosistemas con una diversidad biológica única, la cual sustenta procesos ecológicos esenciales para las poblaciones asentadas al pie de estos. Son, además, parte de los ecosistemas más amenazados por la deforestación y el cambio climático. Sin embargo, a pesar de su importancia y el peligro en el que se encuentran, su comprensión es aún incipiente, siendo las evaluaciones de flora insuficientes en varias zonas de estos bosques (Stadtmüller 1987). 
Diversos factores han contribuido a la pérdida y degradación de estos bosques, los cuales continúan siendo sometidos a procesos de explotación, colonización, deforestación, fragmentación y extracción de recursos no maderables. Algunos de estos factores han sido: el crecimiento de la población, la desigualdad social (pobreza), el establecimiento de cultivos ilícitos, la apertura de nuevas vías de comunicación y la falta de planificación en la expansión de varias actividades como la minería, la extracción de gas y los sistemas agropecuarios (IAASTD 2009, Armenteras et al. 2011, Dávalos et al. 2011, Pacheco et al. 2011). La deforestación en los Andes se debe a una compleja interacción de diferentes fuerzas sociales, culturales, políticas, tecnológicas y económicas que se presentan en la región. Según FAO (2010), la tasa de pérdida total entre los años 2005 y 2010 fue de 6418000 ha de bosque. Entre los países en desarrollo, los Andes constituyen una de las regiones del planeta donde la escasez de leña es más aguda (FAO 1981a, 1983); siendo las regiones altiplánica y altoandina las que registran las mayores penurias, son en estos espacios donde los recursos de leña se han agotado a tal punto que la población ya no está en condiciones de satisfacer sus necesidades mínimas (Ocaña 1994). La importancia de los combustibles tradicionales, en particular la leña y el carbón vegetal, y las graves consecuencias que su escasez entraña desde el punto de vista del aprovisionamiento energético y de la explotación excesiva de los recursos leñosos, hacen necesaria una evaluación de la situación existente, en materia de leña, en los países en desarrollo (FAO 1981b).

Según los reportes oficiales de la FAO (1983), se ha calculado que 1052 millones de personas se hallaban en situación de déficit en lo que se refiere al aprovisionamiento de leña: esas personas no estaban en condiciones de cubrir sus necesidades mínimas a no ser recurriendo a una sobreexplotación que ponía en peligro su aprovisionamiento para el futuro, al no estar asegurada la renovación de los recursos. Además, de acuerdo con los datos oficiales del MINAGRI (2012), en el Perú el consumo de leña y carbón vegetal representa la conversión del $90 \%$ del volumen de madera extraída del país a energía. Si bien se reconoce la imprecisión de las cifras oficiales, estas fueron usadas para estimar las emisiones brutas de la quema de madera en forma de leña y carbón vegetal. De otro lado, el balance nacional de energía, documento preparado por el Ministerio de Energía y Minas (MEM), permite conocer detalladamente la estructura del sector energético nacional, aun cuando no especifica la fuente proveniente del consumo de leña y los cambios respecto al año anterior y determinar el impacto en el ambiente (MEM 2010).

La cordillera del Vilcanota, localizada en el departamento del Cusco, es parte de la cadena oriental de los Andes del Perú, delimitada por los ríos Vilcanota al sur y Yanatile al norte (INAIGEM 2013), el ámbito de estudio constituye uno de los ramales meridionales de la cordillera oriental de los Andes, también denominada la cordillera sagrada pues se halla situada en la margen derecha del río Urubamba, en el Valle Sagrado de los Incas. Los bosques naturales en los Andes son recursos renovables que pueden dar una producción permanente de bienes y servicios ecosistémicos (FAO 1983). Asimismo, MINAGRI (2012), concluye que el uso como material de construcción o fuente de energía para la cocción de alimentos en la vivienda, agota la biomasa vegetal. Las comunidades campesinas de los altos Andes, ubicadas en la cordillera del Vilcanota requieren productos del bosque como fuente de energía, la leña es proveniente de bosques relictos dominados por Escallonia, Polylepis, Gynoxys, Buddleja, Alnus, Citharexylum, Myrcianthes, Weinmannia, Myrica, Duranta, etc; esta demanda agota la oferta de bosques nativos (Gil-Mora et al. 2020) (Cuadro 1).

La demanda de leña por las comunidades campesinas y la oferta de leña por los bosques comunales y otros servicios ambientales brindados por dichos bosques, deben ser conocidos a fin de proponer acciones de protección, manejo y conservación; además el conocimiento de las especies más importantes y requeridas por los campesinos, serán de utilidad para la 
Oferta de leña de los bosques nativos comunales en la cordillera del Vilcanota, Cusco, Perú

\begin{tabular}{|c|c|c|c|c|}
\hline Nombre común & Nombre científico & Familia & Hábito & $\begin{array}{l}\text { Uso como } \\
\text { combustible }\end{array}$ \\
\hline Aliso & Alnus acuminata Kunth & Betulaceae & Árbol & Alto \\
\hline Wichullo & Weinmannia pentaphylla Ruiz \& Pav. & Cunoniaceae & Árbol & Alto \\
\hline P'ispita & Acalypha aronioides Pax \& K. Hoffm. & Euphorbiaceae & Arbusto & Alto \\
\hline Vinagrillo & Sebastiana obtusifolia (H.B.K.) Pax \& K. Hoffm. & Euphorbiaceae & Arbolillo & Alto \\
\hline Vino vino & Aristeguietia discolor R.M king \& H. Rob. & Asteraceae & Arbusto & Bajo \\
\hline Chillca & Baccharis buxifolia (Lam.) Pers. & Asteraceae & Arbusto & Alto \\
\hline Mayu Chilca & Baccharis latifolia (Ruiz \& Pav.) Pers. & Asteraceae & Arbusto & Alto \\
\hline Tayanka & Baccharis odorata Kunth & Asteraceae & Arbusto & Alto \\
\hline Molle & Schinus molle $\mathrm{L}$. & Anacardiaceae & Árbol & Alto \\
\hline China molle & Schinus pearcei Engl. & Anacardiaceae & Arbolillo & Medio \\
\hline Olivero & Pineda incana Ruiz \& Pav. & Salicaceae & Arbusto & Medio \\
\hline Laurel de puna & $\begin{array}{l}\text { Morella pubescens (Humb. Bonpl. ex Willd.) } \\
\text { Wilbur }\end{array}$ & Myricaceae & Árbol & Alto \\
\hline Monte cheqche & Berberis carinata Lechl. & Berberidaceae & Arbusto & Alto \\
\hline Cheqche & Berberis boliviana Lechl. & Berberidaceae & Arbusto & Alto \\
\hline Waka asta & Berberis commutata Eichler & Berberidaceae & Arbolillo & Alto \\
\hline Llaulli & $\begin{array}{l}\text { Barnadesia horrida (Humb. Bonpl ex Willd) } \\
\text { Wilbur }\end{array}$ & Asteraceae & Arbusto & Alto \\
\hline Llaulli & Barnadesia berberoides Sch. Bip. & Asteraceae & Arbusto & Alto \\
\hline Táncar llaulli & Dasyphyllum leiocephalum (Wedd.) Cabrera & Asteraceae & Arbusto & Alto \\
\hline Mot'e mot'e & Duranta mandonii Moldenke & Verbenaceae & Arbolillo & Alto \\
\hline Q'oto kiswar & Gynoxys longifolia Wedd. & Asteraceae & Arbolillo & Alto \\
\hline Qóto kiswar & Gynoxys callacallana Cuatrec. & Asteraceae & Arbusto & Alto \\
\hline Tóqarway & Gynoxys nitida Muschl. & Asteraceae & Árbol & Alto \\
\hline $\begin{array}{l}\text { Kiska mayu } \\
\text { manzana }\end{array}$ & Hesperomeles heterophylla Hook & Rosaceae & Arbusto & Alto \\
\hline Mayu manzana & Hesperomeles lanuginosa Ruiz \& Pav. ex Hook & Rosaceae & Árbol & Alto \\
\hline Lenle & Hesperomeles latifolia (Kunth) M. Roem. & Rosaceae & Árbol & Alto \\
\hline Unca & Myrcianthes oreophilla (Diels) McVaugh & Myrtaceae & Árbol & Alto \\
\hline Maqui maqui & Oreopanax ischnolobus Harms & Araliaceae & Arbusto & Bajo \\
\hline Huamanpinta & Columellia obovata Ruiz \& Pav. & Columelliaceae & Arbusto & Alto \\
\hline Chiñuelas & Saracha punctata Ruiz \& Pav. & Solanaceae & Arbolillo & Alto \\
\hline Ñukao & Cestrum conglomeratum Ruiz \& Pav. & Solanaceae & Arbusto & Medio \\
\hline Tánkar quiska & Lycianthes lycioides (L.) Hassl. & Solanaceae & Arbusto & Alto \\
\hline T'ankar & Dunalia spinosa (Meyen) Dammer & Solanaceae & Arbusto & Alto \\
\hline Chalanque & Myrsine pelucida (Ruiz \& Pav.) Spreng & Primulaceae & Árbol & Medio \\
\hline Thire & Brachyotum naudinii Triana & Melastomataceae & Arbusto & Bajo \\
\hline Chinchircuma & Mutisia acuminata Ruiz \& Pav. & Asteraceae & Arbusto & Medio \\
\hline China Kanlli & Margiricarpus cristatus Britton & Rosaceae & Arbusto & Alto \\
\hline
\end{tabular}

Cuadro 1. Especies utilizadas como leña en comunidades campesinas de la cordillera del Vilcanota. Fuente: Tomado de Gil-Mora et al. 2020. 


\begin{tabular}{|c|c|c|c|c|}
\hline Nombre común & Nombre científico & Familia & Hábito & $\begin{array}{l}\text { Uso como } \\
\text { combustible }\end{array}$ \\
\hline Achupalla & Puya herrerae Harms & Bromeliaceae & Roseta & Bajo \\
\hline Qolle & Buddleja coriácea J. Rémy & Buddlejaceae & Arbolillo & Alto \\
\hline Kiswar-kiswar & Buddleja incana Ruiz \& Pav. & Buddlejaceae & Árbol & Alto \\
\hline Chiqllurmay & Vallea stipularis L.f. & Elaeocarpaceae & Árbol & Alto \\
\hline Kuruchu & Citharexylum argutedentatum Moldenke & Verbenaceae & Árbol & Alto \\
\hline Mutuy & $\begin{array}{l}\text { Senna birostris (Dombey ex Vogel) H.S. Irwin \& } \\
\text { Barneby }\end{array}$ & Fabaceae & Arbusto & Alto \\
\hline Asnaq mutuy & $\begin{array}{l}\text { Senna multiglandulosa (Jacq.) H.S. Irwin \& } \\
\text { Barneby }\end{array}$ & Fabaceae & Arbolillo & Alto \\
\hline Tara & Caesalpinia spinosa (Molina) Kuntze & Escalloniaceae & Árbol & Alto \\
\hline $\begin{array}{l}\text { Qeswa Chacha- } \\
\text { como }\end{array}$ & Escallonia herrerae Mattf. & Escalloniaceae & Árbol & Alto \\
\hline Chachacomo & Escallonia resinosa (Ruiz \& Pav.) Pers. & Escalloniaceae & Árbol & Alto \\
\hline T'asta & Escallonia myrtilloides L.f. & Escalloniaceae & Árbol & Alto \\
\hline Lloque & Kageneckia lanceolata Ruiz \& Pav. & Rosaceae & Arbolillo & Alto \\
\hline Moq'o moq'o & Piper elongatum Vahl & Piperaceae & Arbusto & Bajo \\
\hline Queuña & Polylepis racemosa Ruiz \& Pav. & Rosaceae & Árbol & Alto \\
\hline Queuña & Polylepis incana Kunth & Rosaceae & Árbol & Alto \\
\hline Queuña & Polylepis pauta Hieron. & Rosaceae & Árbol & Alto \\
\hline Queuña & Polylepis subsericans J. F. Macbr. & Rosaceae & Árbol & Alto \\
\hline Queuña & Polylepis sericea Wedd. & Rosaceae & Árbol & Alto \\
\hline Queuña & Polylepis pepei B.B. Simpson & Rosaceae & Arbolillo & Alto \\
\hline Queuña & Polylepis microphylla (Wedd.) Bitter & Rosaceae & Arbusto & Alto \\
\hline
\end{tabular}

Cuadro 1 (continuación). Especies utilizadas como leña en comunidades campesinas de la cordillera del Vilcanota. Fuente: Tomado de Gil-Mora et al. 2020.

gestión de los bosques en los altos Andes. En este contexto, la presente investigación evalúa la oferta de leña que brindan los bosques nativos comunales en los altos Andes ubicados en la cordillera del Vilcanota.

\section{Materiales y Métodos}

\section{Ámbito de Estudio}

Los bosques comunales estudiados se encuentran por encima de los $3200 \mathrm{~m}$ en el flanco oriental de la cordillera del Vilcanota; seis de ellos en la microcuenca del río Patacancha, distrito de Ollantaytambo (provincia de Urubamba) a la que denominamos como “zona 1" (13 ${ }^{\circ} 17^{\prime} 37^{\prime}$ 'S, $\left.72^{\circ} 02^{\prime} 04^{\prime} \mathrm{O}\right)$, y trece en las microcuencas de los ríos Lares, distrito de
Lares y el río Warán, distrito de Calca (ambos en la provincia de Calca) a la que denominamos como “zona 2" (13²'01"S, 7209'27"O) (ver Figura 1); para el estudio, estos bosques han sido divididos en dos pisos altitudinales; aquellos ubicadas por debajo de los $3500 \mathrm{~m}$ y otros por encima de ese nivel altitudinal; la finalidad de esta división es debido a que los bosques comunales ubicados por encima de los $3500 \mathrm{~m}$ poseen escasa oferta de leña, provenientes sustancialmente de bosques de Polylepis.

\section{Metodología}

Para las mensuraciones de la oferta de leña se emplearon transectos lineales y parcelas con la finalidad de determinar la densidad de las especies y obtener información sobre la oferta de 
Oferta de leña de los bosques nativos comunales en la cordillera del Vilcanota, Cusco, Perú

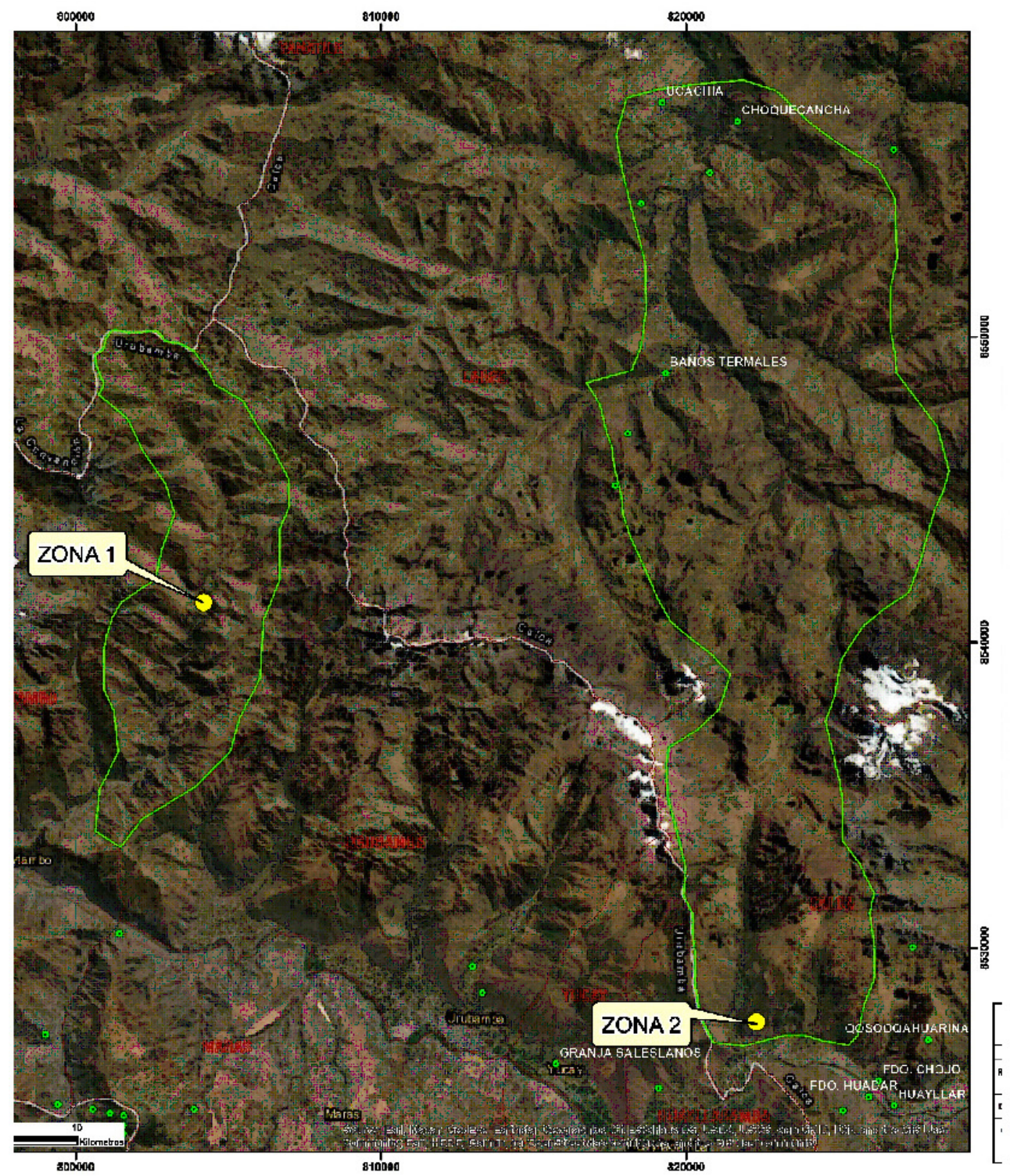

Figura 1. Ámbito de estudio. Ubicación Referencial. Zona 1:3316 m, coordenadas latitud sur 13¹7’37”y latitud oeste $72^{\circ} 02^{\prime} 04^{\prime \prime}$. Zona 2: $4250 \mathrm{~m}$, coordenadas latitud sur $13^{\circ} 12^{\prime} 01^{\prime \prime}$ y latitud oeste $72^{\circ} 09^{\prime} 27^{\prime \prime}$.

leña; para ello, se identificó el territorio en unidades homogéneas, dependiendo del tipo de vegetación a evaluar: para el matorral espinoso, se utilizó el transecto lineal y, para los bosques mixtos y los de Polylepis, la parcela rectangular o cuadrada.
Los transectos lineales utilizados fueron adaptados de Bennet y Humphries (1981) y Franco et al. (1985), con una dimensión de 2 $\mathrm{m}$ de ancho y $25 \mathrm{~m}$ de longitud en razón a la topografía del terreno. Para el registro de datos en campo, se eligieron los transectos lineales 


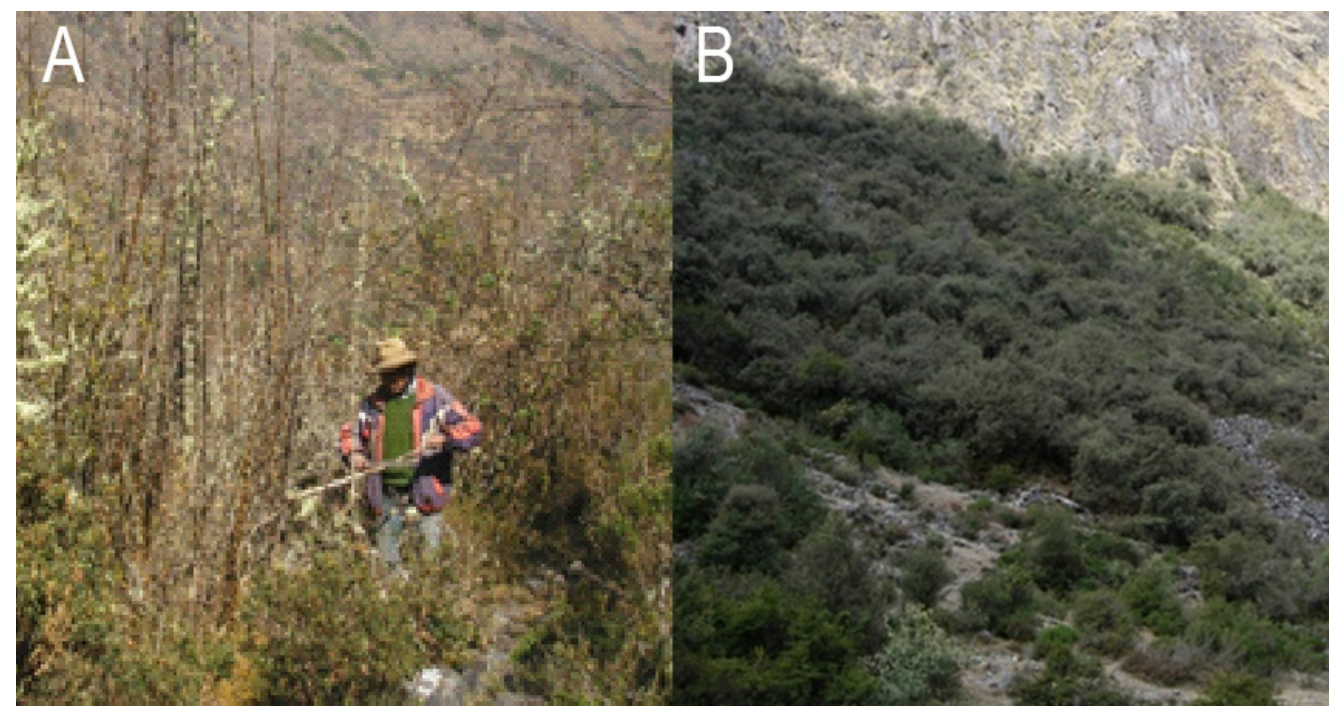

Figura 2. El matorral espinoso y los bosques monoespecíficos de Polylepis constituyen ecosistemas con servicios ambientales importantes para las comunidades campesinas altoandinas.

como unidad muestral por la eficiencia con que pueden ser ejecutados en laderas y por la mayor sensibilidad para detectar la heterogeneidad en la vegetación (Mostacedo y Fredericksen 2000); en total, se utilizaron 32 transectos.

Para delinear la parcela cuadrada o rectangular, dependiendo de la pendiente en el terreno, se adaptó la metodología propuesta por Barbour et al. (1987). En algunos casos, las parcelas fueron diseñadas a favor de la pendiente y otras fueron establecidas horizontalmente, dependiendo de la topografía y la abundancia de la vegetación a mensurar. Se aplicaron 28 parcelas rectangulares y 12 cuadradas de dimensiones $10 \times 20 \mathrm{~m}$ y $10 \times 10 \mathrm{~m}$, respectivamente.

Para la determinación del volumen de leña en los bosques, se mensuraron árboles y arbustos con diámetro superior a $10 \mathrm{~cm}$ a una altura de 1,30 m (FAO 2004, DGFFS 2012, OSINFOR/DGFFS/PRMRFFS 2012), definido como diámetro a la altura del pecho (DAP) utilizado para calcular el volumen del tronco de los árboles (WWF 2006). Y para el caso de los matorrales, se mesuraron todos los arbustos con una circunferencia basal superior a $3 \mathrm{~cm}$. Para el posterior cálculo del volumen y densidad de la madera, en cada individuo registrado se midió la circunferencia basal y distal del fuste, y la longitud del fuste. Con las circunferencias medidas en campo, se determinaron los radios ( $\mathrm{r}$ y R), siendo la explicación técnica la siguiente:

$$
\text { radio }=\frac{L}{2 \pi}
$$

$\mathrm{L}=$ Longitud de la circunferencia basal y distal

El objetivo del uso de esta metodología fue para determinar la densidad ecológica; a partir de los resultados, se extrapolaron para la superficie total de los bosques comunales. Para la determinación del área de los bosques y matorrales, se utilizó la información del proyecto de instalación de plantaciones forestales en áreas no intervenidas en las provincias de Calca y Urubamba (Gobierno Regional del Cusco 2013). La superficie estimada para cada bosque (ha), la densidad ecológica para la totalidad de las especies evaluadas (árboles/ha); constituyen información para estimar la oferta total de leña, medida en toneladas.

El universo para el estudio estuvo conformado por 19 bosques comunales ( 6 en la zona 1 y, 
Oferta de leña de los bosques nativos comunales en la cordillera del Vilcanota, Cusco, Perú

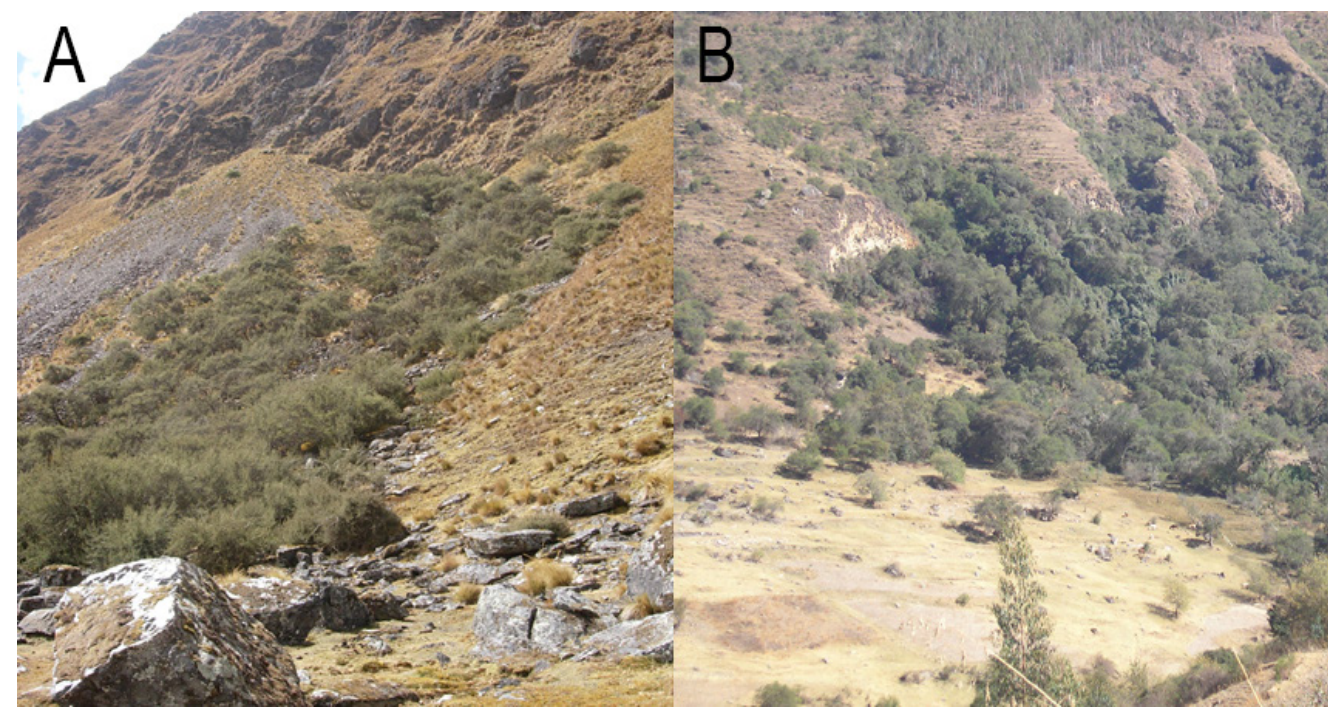

Figura 3. Debido al sobre uso de la cobertura vegetal, hoy ésta se halla muy rala o confinada a oquedales y a las estribaciones de la cordillera.

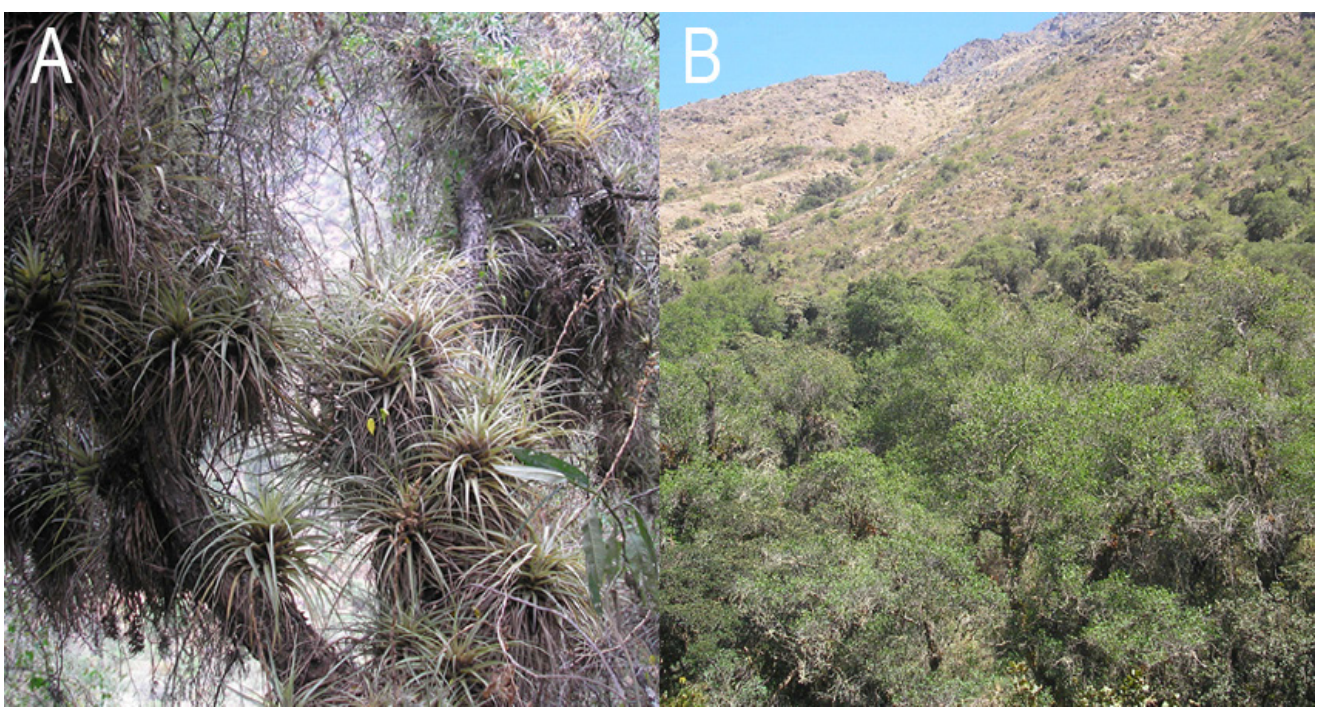

Figura 4. Los bosques mixtos, constituyen ecosistemas que albergan una elevada biodiversidad y recursos genéticos nativos, además de brindar los servicios ambientales. 
13 en la zona 2) y la unidad de análisis para la oferta de leña (biomasa vegetal) fue la tonelada (t) y para la superficie forestal que presentan los bosques nativos fue la hectárea (ha).

\section{Determinación de oferta de leña}

Para la evaluación y posterior estimación de la oferta de biomasa vegetal en los bosques nativos comunales, se consideró que el árbol tiene un fuste cilíndrico truncado, denominado tronco de cono de revolución; determinándose las siguientes variables:

Cálculo del volumen del tronco (leña). El volumen del tronco de cada individuo registrado fue calculado usando la siguiente formula:

$$
\mathrm{V}=\frac{\pi \mathrm{h}}{3}\left(\mathrm{R}^{2}+\mathrm{r}^{2}+\mathrm{Rr}\right)
$$

Donde:

$\pi=3,1416$

$\mathrm{R}$ = radio del cono mayor (calculado a partir de la circunferencia basal)

$\mathrm{r}=$ radio del cono menor (calculado a partir de la circunferencia distal)

$\mathrm{h}=$ altura del tronco de cono de revolución

De acuerdo con estudios realizados por Aróstegui (1974, 1982) y Chambergo (1984), las ramas y otras bifurcaciones leñosas constituyen entre el 15 y el $25 \%$ del volumen o peso total del fuste principal del árbol. Considerando estos estudios, y especialmente la característica fundamental de los árboles y arbustos andinos, que poseen troncos retorcidos, bifurcados y copa ramificada, característica de la vegetación estudiada en las comunidades campesinas, se añadió un $30 \%$ al volumen determinado del fuste principal de las especies estudiadas.

Cálculo para la densidad de la madera. Se colectaron en campo muestras de tallos o troncos de las especies registradas, con una longitud de $50 \mathrm{~cm}$, y en gabinete, se hizo la limpieza obteniéndose muestras del leño de $10 \mathrm{~cm}$ de longitud de diferentes diámetros (de forma similar para el cálculo del contenido de humedad). Posteriormente, se calculó el volumen considerando las medidas de la longitud, de la circunferencia basal y distal. Por otro lado, utilizando una balanza gramera convencional se procedió a determinar la masa de cada muestra recolectada en estado húmedo. Finalmente, con los resultados se procedió a calcular la densidad (D), utilizando la relación entre la masa de la muestra en estado húmedo $(\mathrm{m})$ y su respectivo volumen en estado húmedo (V), conocido como densidad básica y que según Wilson et al. (2007) está definida por la ecuación:

$$
\mathrm{D}=\frac{\mathrm{m}}{\mathrm{V}}\left(\mathrm{kg} / \mathrm{m}^{3}\right)
$$

Contenido de humedad en húmedo base húmeda (M). Viene a ser la masa del contenido de agua en la muestra, la cual es calculada de la diferencia de la masa de la muestra de madera húmeda y la masa de la muestra en estado seco. Dicho procedimiento se efectuó en el laboratorio de calorimetría de la UNSAAC; en campo, se colectaron muestras de tallos o troncos de las especies registradas, con una longitud de 50 $\mathrm{cm}$, y en gabinete, se hizo la limpieza obteniéndose muestras del leño de $10 \mathrm{~cm}$ de longitud de diferentes diámetros, generando tres ejemplares por especie, asimismo, se registró la masa de cada muestra en estado húmedo utilizando una balanza convencional, seguidamente, para el secado de dichas muestras se utilizó una mufla (horno) a $100^{\circ} \mathrm{C}$ durante 72 horas. Finalmente, con los resultados se procedió a calcular el contenido de humedad expresada en porcentaje (M) de los tres ejemplares (Pérez et al. 2011); por lo tanto, y los resultados obtenidos fueron promediados para obtener el valor para cada especie. Para el cálculo del contenido de humedad se utilizó la siguiente expresión:

$$
\mathrm{M}=\frac{\left(\mathrm{m}-\mathrm{m}_{0}\right)}{\mathrm{m}} \times 100 \%
$$

Donde:

$\mathrm{m}=$ masa de la muestra de madera húmeda (gramos)

$\mathrm{m}_{0}=$ masa de la muestra seca (gramos)

Oferta de leña $(t)$. Finalmente, tomando en consideración el volumen del tronco $\left(\mathrm{m}^{3}\right)$ y la densidad de la madera $\left(\mathrm{kg} / \mathrm{m}^{3}\right)$, se calculó la oferta de leña en toneladas para cada especie 


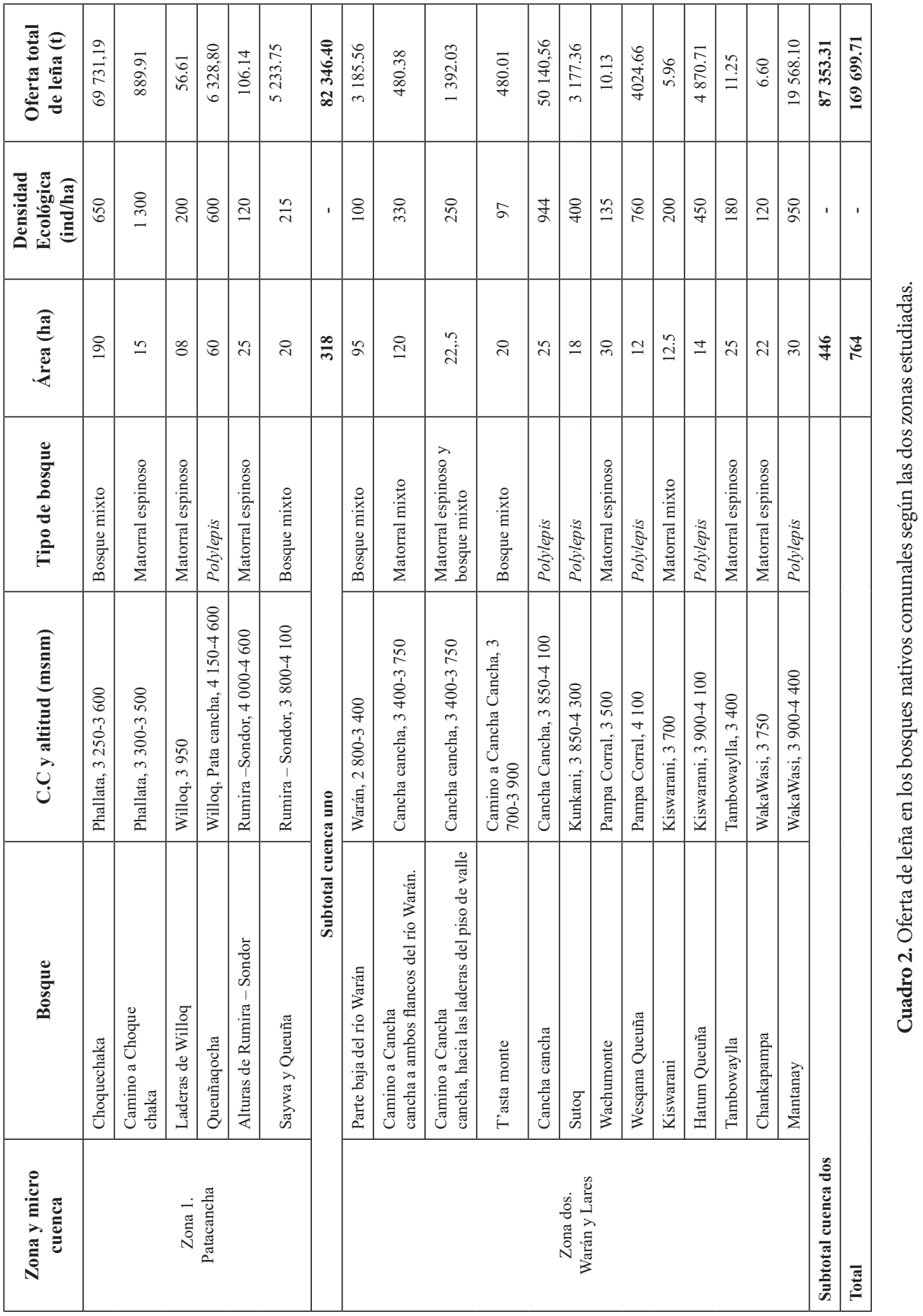


nativa presente en las dos zonas evaluadas, y usando la densidad de individuos se extrapoló la oferta de leña de cada especie para la superficie de todos los bosques comunales en el área de estudio.

\section{Resultados}

El estudio analiza la oferta de leña de los bosques remanentes en los altos Andes utilizados como combustible por las comunidades campesinas en cuya jurisdicción se hallan los 19 bosques nativos comunales distribuidos en una extensión de 764 ha. La zona uno con una superficie de 318 ha posee una oferta de 82346,40 t de leña (48,52 \% del total); en cambio, la zona dos, con un área de 446 ha, presenta una oferta de 87353,31 t de leña $(51,48 \%$ del total). Entre ambas zonas, la oferta de los bosques comunales estudiados para la cordillera del Vilcanota es el equivalente a $169699,71 \mathrm{t}$ de leña (Cuadro 2).

De la superficie total de bosques estudiados, 325 ha $(42,54 \%)$ son bosques mixtos arbóreos y poseen una densidad de 266 individuos/ha y, provisionan 78 630,51 t (46,34\%) de leña; 159 ha $(20,81 \%)$ son bosques de Polylepis, con una densidad de 684 individuos/ha y que ofertan 88 110,19 t (51,92 \%) de leña; finalmente, 280 ha $(36,65 \%)$ corresponden a matorral espinoso, que poseen una densidad de 323 individuos/ha y, oferta 2 959,01 t (1,74\%) de leña (ver Cuadros 3,4 y 5 ).

Existe mayor diversidad en los bosques y bosquetes inferiores a $3500 \mathrm{~m}$ constituyendo bosques mixtos, matorrales espinosos y asociaciones interespecíficas; en cambio a altitudes superiores a los $3500 \mathrm{~m}$ se observan rodales dominados por los géneros Polylepis, Gynoxis, Weinmannia, Cytharexilum y Buddleja.

De las especies más utilizadas en el abastecimiento de leña, destacan por su abundancia los géneros Hesperomeles, Buddleja, Escallonia y Polylepis; sin embargo, considerando la biomasa que ofertan, las especies más importantes pertenecen a los géneros Alnus, Acalypha, Baccharis, Myrica, Berberis, Duranta, Barnadesia,
Gynoxis, Hesperomeles, Myrcianthes, Citharexylum, Escallonia y Polylepis (Cuadros 2 y 3 ).

El matorral espinoso es importante, el camefitismo (plantas perennes, leñosas o herbáceas, cuyas ramas tienen la capacidad de rebrote) de las especies que posibilita el rebrote y ramificación exuberante, facilitando la obtención de biomasa vegetal como combustible. Los bosques mixtos y matorrales, con 605 ha, constituyen la zona de mayor oferta de leña, y en altitudes inferiores a $3500 \mathrm{~m}$ poseen una alta productividad y densidad permitiendo que las comunidades campesinas se provean de leña.

Los bosque andinos son de vital importancia por que contribuyen a reducir la vulnerabilidad de los ecosistemas e incrementar resiliencia, contribuir con la adaptación al cambio climático, pues, además de ser reservorios de carbono, desempeñan un rol clave en la provisión de bienes y servicios ecosistémicos, no sólo regulan el clima, suministran agua, frenan inundaciones y sequías y mantienen los hábitats que posibilitan la supervivencia de la biodiversidad en el largo plazo; de modo tal que los bosques andinos gestionados de manera sostenible juegan un rol importante en la regulación hídrica y estabilización del clima; en razón a ello, estos bosques, constituyen el mejor ecosistema forestal, por las especies y por una gradación altitudinal secuencial en la distribución espacial de las especies: Alnus acuminata Hunt, Hesperomeles latifolia (Kunth) M. Roem, Escallonia herrerae Mattf, Myrcianthes oreophila (Diels) Mc Vaugh, Hesperomeles heterophylla Hook, Vallea stipularis L.f, Piper elongatum Vahl, Citharexylum argutedentatum Moldenke, Duranta mandonii Moldenke, Escallonia myrtilloides L.f., Gynoxys nítida Muschl. y Polylepis spp.

\section{Discusión}

Salas (2009), concluye que la extracción de leña y productos forestales no maderables de los bosques nativos andinos de Pacobamba (Apurímac) está muy vinculada con la mujer, mientras que la extracción de madera del bosque nativo es practicada más por los 


\begin{tabular}{|c|c|c|c|c|c|c|c|c|}
\hline Zona & $\begin{array}{l}\text { Formación } \\
\text { vegetal }\end{array}$ & Especie & 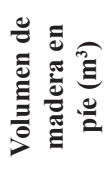 & 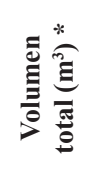 & 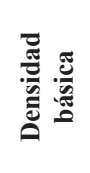 & 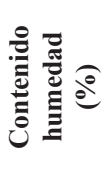 & 氬 & 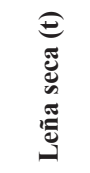 \\
\hline \multirow{11}{*}{$\begin{array}{l}\text { Zona } \\
\text { Uno }\end{array}$} & \multirow{4}{*}{ Bosque mixto } & Myrcianthes oreophylla & 0.2472 & 0.3221 & 0.7900 & 17.1900 & 0.2545 & 0.2102 \\
\hline & & Escallonia herrerae & 1.2975 & 1.6867 & 0.7950 & 36.2100 & 1.3410 & 0.8554 \\
\hline & & Alnus acuminata & 2.3125 & 3.0062 & 0.4700 & 20.1450 & 1.4129 & 1.1283 \\
\hline & & Hesperomeles latifolia & 0.0840 & 0.1092 & 0.7600 & 23.0650 & 0.0830 & 0.0638 \\
\hline & \multirow{6}{*}{$\begin{array}{l}\text { Matorral } \\
\text { espinoso }\end{array}$} & Barnadesia berberoides & 0.0048 & 0.0062 & 0.6700 & 52.8450 & 0.0042 & 0.0020 \\
\hline & & Berberis carinata & 0.0019 & 0.0025 & 0.8500 & 52.1700 & 0.0021 & 0.0010 \\
\hline & & Berberis commutata & 0.0007 & 0.0010 & 0.8500 & 52.1700 & 0.0009 & 0.0004 \\
\hline & & Senna multiglandulosa & 0.0049 & 0.0064 & 0.5600 & 38.9650 & 0.0036 & 0.0022 \\
\hline & & Duranta mandonii & 0.1086 & 0.1412 & 0.5300 & 12.3550 & 0.0748 & 0.0656 \\
\hline & & Baccharis odorata & 0.0064 & 0.0083 & 0.5300 & 30.8800 & 0.0044 & 0.0030 \\
\hline & Polylepis & Polylepis spp. & 0.4554 & 0.5920 & 0.6500 & 30.6450 & 0.3848 & 0.2669 \\
\hline \multirow{20}{*}{$\begin{array}{l}\text { Zona } \\
\text { Dos }\end{array}$} & \multirow{9}{*}{ Bosque mixto } & $\begin{array}{l}\text { Citharexylum } \\
\text { argutedentatum }\end{array}$ & 0.0108 & 0.0140 & 0.8200 & 44.2300 & 0.0115 & 0.0064 \\
\hline & & Escallonia herrerae & 0.9590 & 1.2467 & 0.7950 & 36.2100 & 0.9911 & 0.6322 \\
\hline & & Myrica pubescens & 0.4997 & 0.6496 & 0.6300 & 49.7300 & 0.4093 & 0.2057 \\
\hline & & Escallonia mirtylloides & 0.3824 & 0.4971 & 0.6667 & 44.6600 & 0.3314 & 0.1834 \\
\hline & & Vallea stipularis & 0.0219 & 0.0285 & 0.6100 & 53.2900 & 0.0174 & 0.0081 \\
\hline & & Weinmania pentaphylla & 0.3167 & 0.4117 & 0.5600 & 53.8200 & 0.2306 & 0.1065 \\
\hline & & Gynoxis nitida & 0.2495 & 0.3243 & 0.6900 & 36.7350 & 0.2238 & 0.1416 \\
\hline & & Brachiotum naudinii & 0.0067 & 0.0087 & 0.6100 & 49.6350 & 0.0053 & 0.0027 \\
\hline & & $\begin{array}{l}\text { Hesperomeles } \\
\text { lanuginosa }\end{array}$ & 0.0921 & 0.0276 & 0.7905 & 28.4532 & 0.0218 & 0.0150 \\
\hline & \multirow{10}{*}{$\begin{array}{l}\text { Matorral } \\
\text { espinoso }\end{array}$} & Baccharis odorata & 0.0064 & 0.0083 & 0.5300 & 30.8800 & 0.0044 & 0.0030 \\
\hline & & Berberis spp. & 0.0140 & 0.0182 & 0.8500 & 52.1700 & 0.0155 & 0.0074 \\
\hline & & Berberis carinata & 0.0019 & 0.0025 & 0.8500 & 52.1700 & 0.0021 & 0.0010 \\
\hline & & Berberis commutata & 0.0007 & 0.0010 & 0.8500 & 52.1700 & 0.0009 & 0.0004 \\
\hline & & Weinmania pentaphylla & 0.3167 & 0.4117 & 0.5600 & 53.8200 & 0.2306 & 0.1065 \\
\hline & & Duranta mandonii & 0.1086 & 0.1412 & 0.5300 & 12.3550 & 0.0748 & 0.0656 \\
\hline & & Barnadesia berberoides & 0.0048 & 0.0062 & 0.6700 & 52.8450 & 0.0042 & 0.0020 \\
\hline & & Saracha punctata & 0.0103 & 0.0134 & 0.6300 & 11.9000 & 0.0084 & 0.0074 \\
\hline & & Senna multiglandulosa & 0.0049 & 0.0064 & 0.5600 & 38.9650 & 0.0036 & 0.0022 \\
\hline & & Siphocampilus sp. & 0.0172 & 0.0224 & 0.5400 & 64.5550 & 0.0121 & 0.0043 \\
\hline & Polylepis & Polylepis spp. & 3.6252 & 4.7128 & 0.6500 & 30.6450 & 3.0633 & 2.1246 \\
\hline
\end{tabular}

Cuadro 3. Volúmenes de madera fresca y seca y cálculo de oferta de leña de los bosques nativos comunales en el ámbito de estudio, valores promedio. ${ }^{*}$ ) Para calcular la biomasa leñosa total, se añadió un $30 \%$ al volumen determinado, de acuerdo a estudios realizados por Aróstegui $(1974,1982)$ y Chambergo (1984). 


\begin{tabular}{|c|c|c|c|c|c|}
\hline Tipo de bosque & $\begin{array}{c}\text { Área total } \\
\text { (ha) }\end{array}$ & $\begin{array}{c}\text { Porcentaje } \\
\text { (\%) }\end{array}$ & $\begin{array}{c}\text { Producción de } \\
\text { leña (t) }\end{array}$ & Porcentaje (\%) & $\begin{array}{c}\text { Oferta leña (t/ } \\
\text { ha) }\end{array}$ \\
\hline Mixto arbóreo & 325 & 42.54 & 78630.51 & 46.34 & 241.94 \\
\hline Matorral espinoso & 280 & 36.65 & 2959.01 & 01.74 & 10.57 \\
\hline Polylepis & 159 & 20.81 & 88110.19 & 51.92 & 554.15 \\
\hline Total & $\mathbf{7 6 4}$ & $\mathbf{1 0 0 . 0 0}$ & $\mathbf{1 6 9} \mathbf{6 9 9 . 7 1}$ & $\mathbf{1 0 0 . 0 0}$ & $\mathbf{2 2 2 . 1 2}$ \\
\hline
\end{tabular}

Cuadro 4. Producción de leña, según tipo de bosque y oferta leña (t/ha).

\begin{tabular}{|c|c|c|c|c|c|c|}
\hline Zona & $\begin{array}{c}\mathbf{N}^{\circ} \text { Comunidades } \\
\text { campesinas }\end{array}$ & $\begin{array}{c}\mathbf{N}^{\circ} \text { de } \\
\text { bosques }\end{array}$ & $\begin{array}{c}\text { Área de } \\
\text { bosques (ha) }\end{array}$ & $\begin{array}{c}\text { Porcentaje } \\
\mathbf{( \% )}\end{array}$ & $\begin{array}{c}\text { Oferta de } \\
\text { leña (t) }\end{array}$ & $\begin{array}{c}\text { Porcentaje } \\
(\mathbf{\%})\end{array}$ \\
\hline Uno & 04 & 06 & 318.0 & 41.62 & 82346.40 & 48.52 \\
\hline Dos & 07 & 13 & 446.0 & 58.38 & 87353.31 & 51.48 \\
\hline Total & $\mathbf{1 1}$ & $\mathbf{1 9}$ & $\mathbf{7 6 4}$ & $\mathbf{1 0 0 . 0 0}$ & $\mathbf{1 6 9} 699.71$ & $\mathbf{1 0 0 . 0 0}$ \\
\hline
\end{tabular}

Cuadro 5. Oferta de leña según zonas de estudio.

varones. Además, este estudio sólo recoge información sobre especies vinculadas a la obtención de leña, más no la oferta; nuestro trabajo evidencia la oferta de leña ( $\mathrm{t} / \mathrm{ha})$; en efecto, el bosque mixto ofrece 241,94 t/ha y, el bosque de Polylepis es altamente productivo, pues su oferta alcanza 554,15 t/ha; el matorral espinoso, puede llegar a ofertar hasta 10,47 t/ha; esta oferta es de interés para plantear estrategias de conservación mediante reforestación (ver Cuadro 4).

Los estudios de Córdova (2012), señalan que la oferta de leña en los bosques de las comunidades del bajo Urubamba es alta (100\% de la leña proviene de bosque aledaños, pues el consumo promedio de leña por familia es de 0,125 $\mathrm{m}^{3}$ diarios y $45,6 \mathrm{~m}^{3}$ anuales) y no requieren almacenarla, excepto para actividades comunales; en cambio, en las comunidades altoandinas, se verificó que las familias almacenan la leña para un periodo mayor a una semana; igualmente, el estudio de Córdova (2012), concluye que no es un problema significativo el abastecimiento de leña; en cambio, en las comunidades altoandinas, la oferta de leña proveniente de bosques nativos va disminuyendo, especialmente por encima de los $3500 \mathrm{~m}$.

Los bosques nativos en los altos Andes no sólo están siendo afectados por la sobre ex- tracción de leña como sostiene MINAGRI (2012); el presente estudio ratifica esta conclusión y, además, indica otras connotaciones ambientales que afectan los servicios ecosistémicos. Análogamente a lo reportado por Ocaña (1994), en las comunidades estudiadas que se hallan ubicadas por encima de los 3600 o $4000 \mathrm{~m}$ el déficit de material energético es crítico y no llega a abastecer las necesidades más urgentes, especialmente si de leña se trata; por lo tanto, es substituido por otros elementos como la bosta de ganado (vacuno, ovino y camélidos sudamericanos) generados en la propia comunidad y/o la leña de eucalipto, proveniente de otras zonas.

Desde hace décadas se observa la creciente escasez de leña para uso doméstico, tal como lo sostienen Córdova-Aguilar (1992), Ektvedt (2011) y MINAGRI (2012); en efecto, esta conclusión es ratificada por nuestros trabajos de campo y especifican una oferta escasa de leña de los bosques altoandinos, especialmente para comunidades ubicadas por encima de los 3500 $\mathrm{m}$.

Según sostiene Bozovich (2016), citado por PCM (2021), en el Perú, el 87 \% de la deforestación es causada por la agricultura migratoria de pequeña escala y sus prácticas de rozo y quema y la ganadería extensiva, mientras que 

en la cordillera del Vilcanota, Cusco, Perú

el $8 \%$ por generación de energía (consumo de leña de los hogares rurales) y el $5 \%$ por extracción forestal. Cifras parecidas presentan Galarza y La Serna (2005); igualmente, Torres (2010) sostiene que la agricultura migratoria de subsistencia, principalmente asociada a familias rurales pobres, explica más del $80 \%$ de la deforestación en nuestro país, explicando que la mayor deforestación es por la quema de bosques para agricultura. En la zona andina la agricultura migratoria también tiene intervención sobre los bosques; sin embargo, no son coincidentes con estas cifras. En la cordillera del Vilcanota, la obtención de leña y madera por las comunidades campesinas son las acciones que más contribuyen en la deforestación $\mathrm{y}$ deterioro de los bosques nativos.

Toivonen y Kessler (2006) sostienen que los bosques de Polylepis representan la vegetación natural de una gran parte de los Andes centrales a altitudes entre 3500 y $4400 \mathrm{~m}$, pero en la actualidad se encuentran altamente fragmentados en todas partes de su rango de distribución natural en los Andes, desde Venezuela hasta Argentina. Sin embargo, en la cordillera del Vilcanota hemos registrado bosques de Polylepis a más de $4800 \mathrm{~m}$, de altitud y además siendo los que mayor oferta de leña presentan en el área.

\section{Conclusiones}

Los bosques nativos en los altos Andes tienen especial importancia por la oferta de leña y por la función que desempeña este combustible en la satisfacción de necesidades energéticas tan esenciales como la cocción de alimentos o la calefacción en las viviendas rurales. Su escasez se traduce, en mayores dificultades para subsistir y en una ruptura de sus sistemas energéticos; en los casos extremos, trae consigo una desestabilización del ambiente, debido a la desaparición de los bosques y a la corta de la vegetación leñosa.

El problema de la oferta de leña tiene, tres dimensiones importantes: forestal, energética y ecológica. Por lo tanto, los bosques son fuentes de recursos para las comunidades; sin embargo, actividades como la extracción de leña y made- ra sin reemplazo, la quema para la agricultura, que deviene en incendios, la introducción de ganado exótico y otros, tienen efectos directos en los bosques que son parches aislados. Estas acciones afectan la cobertura vegetal, perturbación que ocasiona impactos ambientales como mayor escorrentía, erosión, disminución de infiltración del agua de precipitación, pérdida de biodiversidad, etc. Es preciso resaltar que las comunidades campesinas altoandinas poseen predisposición para la reforestación y están dispuestos a participar en programas forestales.

En síntesis, la cocción de alimentos y la calefacción de la vivienda representan para las comunidades altoandinas de la cordillera del Vilcanota, la parte más importante del consumo energético total, especialmente en los hogares pobres. La leña es el combustible preferido de las comunidades campesinas de los altos Andes, cuyo acceso a otras fuentes de energía es, muy limitado; por lo tanto, la leña, desempeña una función esencial para atender necesidades energéticas elementales, ligadas a la subsistencia misma de esas poblaciones. La leña tiene una función preponderante en el aprovisionamiento energético de las comunidades altoandinas. En los sistemas energéticos rurales, la leña ocupa un lugar especial, debido a la importancia del consumo doméstico de energía, al que la leña se destina y por el hecho de que se produce dentro mismo del sistema. La oferta total de leña calculada para los bosques altoandinos de la cordillera del Vilcanota es de $169699,71 \mathrm{t}$, con una producción promedio de $222,12 \mathrm{t} / \mathrm{ha}$.

\section{Agradecimientos}

A Ecosistemas Andinos-ECOAN, por posibilitar la ejecución del estudio y, al colega Alfredo Tupayachi Herrera por el apoyo en la identificación de las especies botánicas.

\section{Referencias}

Armenteras, D; Rodríguez, N; Retana, J; Morales, M. 2011. Understanding deforestation 
in montane and lowland forests of the Colombian Andes. Regional Environmental Change 11(3):693-705.

Aróstegui, A. 1974. Estudio tecnológico de maderas del Perú: características tecnológicas y usos de la madera de 145 especies del país. Lima, Perú, Ministerio de Agricultura. 483 p.

Aróstegui, A. 1982. Recopilación y análisis de estudios tecnológicos de maderas peruanas. Lima, Perú. 57 p. Documento de trabajo n. ${ }^{\circ} 2$. Proyecto PNUD/FAO/PER/81/002.

Barbour, MG; Burk, JH; Pitts, WD. 1987. Terrestrial plant ecology. 2 ed. California, Estados Unidos de América, Benjamin/Cummings Publishing Company. 634 p.

Bennet, DP; Humphries, DA. 1981. Introducción a la ecología de campo. Madrid, España, Blume. 322 p.

Chambergo, A. 1984. Influencia de los elementos xilemáticos en las propiedades físico-mecánicas de 45 maderas del Perú. Tesis Ing. Lima, Perú, UNALM. 110 p.

Córdova-Aguilar, H. 1992. Firewood use and the effect on the ecosystem: a case study of the sierra of Piura, northwestern Peru. GeoJournal 26(3):297-309.

Córdova, P. 2012. Estudio del consumo de leña en dos comunidades nativas de la cuenca del rio Bajo Urubamba. Tesis Ing. Lima, Perú, UNALM. 73 p.

Dávalos, LM; Bejarano, AC; Hall, MA; Correa, HL; Corthals, A; Espejo, OJ. 2011. Forests and drugs: coca-driven deforestation in tropical biodiversity hotspots. Environmental Science and Technology 45(4):1219-1227.

DGFFS (Dirección General Forestal y de Fauna Silvestre, Perú). 2012. Manual base para la planificación y ejecución de inventarios forestales. Lima, Perú, MINAGRI. 52 p.

Ektvedt, TM. 2011. Firewood consumption amongst poor inhabitants in a semiarid tropical forest: a case study from Piura, northern Peru. Norsk Geografisk Tidsskrift - Norwegian Journal of Geography 65(1):28-41.
FAO (Organización de las Naciones Unidas para la Alimentación y la Agricultura, Italia). 1981a. (Informe). Conferencia de las Naciones Unidas sobre fuentes de energía nuevas y renovables (1, 1981, Nairobi, Kenia). Roma, Italia.

FAO (Organización de las Naciones Unidas para la Alimentación y la Agricultura, Italia). 1981b. Situación en materia de leña en los países en desarrollo. Roma, Italia.

FAO (Organización de las Naciones Unidas para la Alimentación y la Agricultura, Italia). 1983. Disponibilidad de leña en los países en desarrollo (en línea). Roma, Italia. (Estudio FAO: Montes, n. $\left.{ }^{\circ} 4\right)$. Consultado 12 oct. 2016. Disponible en www.fao.org/3/x5329s/ x5329s02.htm.

FAO (Organización de las Naciones Unidas para la Alimentación y la Agricultura, Italia). 2004. Inventario forestal nacional: manual de campo modelo. Ciudad de Guatemala, Guatemala. $89 \mathrm{p}$.

FAO (Organización de las Naciones Unidas para la Alimentación y la Agricultura, Italia). 2010. La gestión de los bosques ante el cambio climático. Roma, Italia. 19 p.

Franco, F; de la Cruz, A; Rocha, N; Navarrete, G; Flores, E; Kato, S; Sánchez, L; Abarca, LG; Bedia, C. 1985. Manual de ecología. 2 ed. México DF, México, Trillas. 266 p.

Galarza, E; La Serna, C. 2005. Las concesiones forestales en el Perú: ¿cómo hacerlas sostenibles? In Barrantes, $\mathrm{R}$ (ed.). La política forestal en la Amazonía andina, estudio de casos: Bolivia, Ecuador y Perú. Lima, Perú, CIES.p. 445-600. (Serie Diagnóstico y Propuesta, n. $\left.{ }^{\circ} 18\right)$

Gil-Mora, JE; Baca-Flores, JA; Álvarez-Moscoso, ME. 2020. Consumo de leña y estimaciones del valor calorífico de especies de plantas utilizadas por las comunidades campesinas de la cordillera del Vilcanota, Cusco. Revista Q'euña 11(1):41-58. DOI: https://doi.org/10.51343/ rq.v11i1.431.

Gobierno Regional del Cusco. 2013. Proyecto de instalación de plantaciones forestales en áreas no intervenidas en las provincias de 

en la cordillera del Vilcanota, Cusco, Perú

Calca y Urubamba de la región Cusco. Cusco, Perú, AFEP. 96 p.

IAASTD (International Assessment of Agricultural knowledge, Science and Technology for Development, Estados Unidos de América). 2009. Evaluación internacional del papel del conocimiento, la ciencia y la tecnología en el desarrollo agrícola. Washington DC, Estados Unidos de América. 45 p.

INAIGEM (Instituto Nacional de Investigación en Glaciares y Ecosistemas de Montaña, Perú). 2013. Estudios glaciológicos en la cordillera Vilcanota. Huaraz, Perú. s.p.

MINAGRI (Ministerio de Agricultura y Riego, Perú). 2012. Perú forestal en números (en línea). Lima, Perú. 164 p. Consultado 5 jun. 2012. Disponible en http://dgffs.minag.gob.pe/ pdf/estadistica forestal/anuarios/ANUARIO PERU FORESTAL 2011.pdf.

MEM (Ministerio de Energía y Minas, Perú). 2010. Balance nacional de energía (en línea). Lima, Perú. Consultado 20 may. 2014. Disponible en http://www.minem.gob.pe/publicacion.php?idSector=12\&idPublicacion=418.

Mostacedo, B; Fredericksen, TS. 2000. Manual de métodos básicos de muestreo y análisis en ecología vegetal. Santa Cruz, Bolivia, BOLFOR. $87 \mathrm{p}$.

Ocaña, D. 1994. Desarrollo forestal campesino en la región andina del Perú. Lima, Perú, Ministerio de Agricultura. 218 p.

OSINFOR (Organismo de Supervisión de los Recursos Forestales y de Fauna Silvestre, Perú) / DGFFS (Dirección General Forestal y de Fauna Silvestre, Perú) / PRMRFFS (Programa Regional de Manejo de Recursos Forestales y de Fauna Silvestre, Perú). 2012. Protocolo para la evaluación de individuos maderables: proceso de convergencia metodológica interinstitucional para la estandarización de los criterios de evaluación de recursos forestales maderables en bosques húmedos. Lima, Perú. 13 p.

Otárola, E. 2008. Analizando las causas subyacentes de la deforestación y sus posibles implicancias para REDD. Lima, Perú, WWF. 47 p.
Pacheco, C; Aguado, I; Mollicone, D. 2011. Las causas de la deforestación en Venezuela: un estudio retrospectivo. BioLlania (10):281-292.

PCM (Presidencia del Consejo de Ministros, Perú). 2021. Buenas prácticas: casos destacados en manejo forestal sostenible en la Amazonía peruana. Lima, Perú. 78 p. (Colección Estimando y mejorando la legalidad de la madera en el Perú, n. ${ }^{\circ}$ 6).

Pérez, N; Valenzuela, L; Diaz, JE; Ananías, RA. 2011. Predicción del contenido de humedad de equilibrio de la madera en función del peso específico de la pared celular y variables ambientales. Maderas, Ciencia y tecnología 13(3):253-266. DOI: https://doi.org/10.4067/ S0718-221X2011000300002.

Salas, R. 2009. Diagnóstico con enfoque de género sobre el uso de los bosques nativos andinos de Pacobamba-Apurímac. Tesis Ing. Lima, Perú, UNALM. 127 p.

Stadtmüller, T. 1987. Los bosques nublados en el trópico húmedo: una revisión bibliográfica. San José, Costa Rica, CATIE. 85 p.

Toivonen, J; Kessler, M. 2006. Distribución geográfica y los nichos ocupados de los bosques actuales de Polylepis en la región del Cusco, Perú. Congreso de Ecología y Conservación de Bosques de Polylepis. (2, 2006, Cusco, Perú). Cusco, Perú.

Torres, JA. 2010. Herramientas económicas para la conservación del bosque amazónico: avances y limitaciones. Tesis Lic. Lima, Perú, PUCP. 89 p.

Wilson, JD; Buffa, AJ; Lou, B. 2007. Física. 6 ed. Ciudad de México, México, Pearson Prentice Hall. 912 p.

WWF (World Wildlife Fund, Suiza). 2006. Manual de censos forestales. Lima, Perú. 72 p.

Young, K; León, B. 2001. Perú. In Kappelle, M; Brown, A (eds.). Bosques nublados del Neotrópico. San José, Costa Rica, Instituto Nacional de Biodiversidad. p. 549-580. 